\title{
Diagnóstico precoce da hanseníase: o caso dos serviços de saúde no Recife (Pernambuco), Brasil ${ }^{1}$
}

\author{
Katia V. de O. Feliciano, ${ }^{2}$ Maria Helena Kovacs ${ }^{2}$ e Alberto Alzate ${ }^{3}$
}

RESUMEN O presente trabalho é um estudo descritivo, realizado na cidade do Recife de março a setembro de 1994. O estudo buscou caracterizar as condições existentes nos serviços de saúde para o diagnóstico precoce de hanseníase, com ênfase na acessibilidade dos usuários aos serviços e na qualidade do atendimento. A amostra constou de 32 serviços de saúde utilizados durante o processo diagnóstico de 183 pacientes de hanseníase. A infra-estrutura organizacional foi caracterizada através de entrevistas com os gerentes dos serviços. O funcionamento foi investigado através de observações da rotina dos serviços, em particular as atividades de arquivo e triagem. Um total de 1998 pacientes foram entrevistados para determinar a facilidade de acesso. Para 1000 pacientes foi estabelecido o tempo de consulta com os 123 médicos que estavam nas unidades no momento da amostragem. Para explorar a atitude e o conhecimento dos médicos sobre a hanseníase, selecionou-se, aleatoriamente, a partir de uma listagem nominal, 133 médicos destes serviços.

Identificaram-se como dificuldades para o diagnóstico precoce de hanseníase: a sistemática demanda reprimida (pessoas que procuravam os serviços mas não eram atendidas); o longo tempo entre momento de marcação e momento de realização de consultas (para pessoas que não eram atendidas no mesmo dia); os longos tempos de espera pela consulta dentro dos serviços; a curta duração da consulta; a baixa disponibilidade de recursos humanos treinados; a baixa proporção de médicos que examinam toda a superfície corporal; as deficiências no reconhecimento clínico da doença e no conhecimento que leva ao diagnóstico diferencial. Estes obstáculos podem facilitar a deterioração física dos portadores de hanseníase e a persistência da transmissibilidade; portanto, necessitam ser superados para que seja possível eliminar a hanseníase.

Na cidade do Recife, Brasil, registrou-se um crescimento das taxas de

\footnotetext{
Esta investigação foi financiada através do Programa Especial para Pesquisa e Treinamento em Doenças Tropicais (TDR), Programa das Nações Unidas para o Desenvolvimento/Banco Mundial/ Organização Mundial da Saúde (Projeto ID920523).

2 Universidade de Pernambuco, Fundação de Ensino Superior de Pernambuco, Faculdade de Ciências Médicas de Pernambuco, Pernambuco, Brasil. Correspondência e pedidos de separatas devem ser enviados a Katia V. de O. Feliciano no seguinte endereço: Rua Arnóbio Marques 310, Santo Amaro, CEP 50100-130, Recife, PE, Brasil. Fax: +5581-423-33-71.

3 Universidade do Valle, Faculdade de Saúde, Cali, Colômbia.
}

detecção das formas clínicas tuberculóide e dimorfa da hanseníase $(1,2)$, para ambos os sexos e independente da idade. Tal fato sugere um aumento no risco de desenvolvimento precoce de incapacidades provocadas pela doença, assim como de ocorrência de quadros reacionais.

O diagnóstico precoce e o tratamento adequado dos portadores de hanseníase são condições essenciais para interromper a transmissão e reduzir as conseqüências físicas e sociais da doença $(3,4)$; o tratamento é facilitado atualmente pela disponibilidade de drogas eficazes, de fácil aplicação e baixo custo, e de técnicas simples para a prevenção das incapacidades provocadas pela hanseníase, assim como pela prática de auto-cuidado pelos portadores, que deve ser estimulada. Além do mais, alguns fatores apontam para a possibilidade prática de eliminação da hanseníase (5), como por exemplo: a) características clínicas e epidemiológicas que permitem a detecção oportuna dos casos; b) o reconhecimento, nacional e internacional, da importância socioeconômica da doença e c) a emergência de resistência primária 
(6), uma razão específica para preferir a sua eliminação e não o seu controle.

Após analisar alguns indicadores do comportamento da hanseníase e da operacionalização das atividades de controle da doença durante o período de 1987 a 1993, o Ministério da Saúde do Brasil, através da coordenação nacional de dermatologia sanitária, considerou, como condições propícias para a eliminação da doença no Brasil, a semelhança entre o número de casos estimados e detectados, a tendência ao declínio da prevalência da doença, a redução da proporção de incapacidades entre os casos novos, o crescimento da cobertura de poliquimioterapia e o grande número de técnicos que tinham sido capacitados (7).

A estratégia nacional de eliminação da doença, com o propósito de reduzir a prevalência da hanseníase no país para menos de 1/10 000 habitantes até o ano 2000, após a etapa preparatória, está sendo desenvolvida em três fases principais: 1) a fase intensiva - adoção de medidas para interromper a transmissão; 2) a fase de consolidação - localização e eliminação dos casos residuais e importados e 3) a fase de manutenção - manutenção dos resultados obtidos mediante uma cuidadosa vigilância. O estado de Pernambuco, desde 1995, está alocado na etapa final da fase intensiva.

Estudos sobre a transmissão da hanseníase demonstram que as pessoas que convivem e têm contato com os doentes estão expostas a um maior risco de adoecer quando comparadas com a população geral. Os contatos domiciliares de casos multibacilares representam um grupo caracterizado por altas taxas de ataque $(4,6)$. Mesmo assim, em áreas de alta prevalência da doença, considera-se que mais da metade dos casos são diagnosticados entre a população em geral (6). Isto indica que a detecção da hanseníase nas consultas em clínicas gerais e dermatológicas da rede de saúde é fundamental para interromper a transmissão da doença e para evitar a deterioração física dos seus portadores.

Em uma conjuntura específica, o modo como são produzidas as ações de saúde e a maneira como os serviços se organizam para produzi-las e distribuí-las são resultantes das diversas políticas de confronto de interesses (8). Sendo a população múltipla em suas demandas, em uma rede insuficiente e subutilizada, muitas dificuldades se interpõem, no dia-a-dia, entre a disponibilidade planejada e a utilização efetiva dos serviços de saúde.

Por outro lado, o manejo da hanseníase dentro da rede de serviços de saúde encontra fortes obstáculos técnicos, administrativos e organizacionais, comumente encontrados na descentralização das ações de controle de doenças específicas, como tuberculose, leishmaniose e raiva (9). A estes obstáculos se somam o preconceito existente contra os portadores da hanseníase e o medo que esta doença provoca entre profissionais do setor de saúde (10).

Devemos compreender que a efetividade de toda e qualquer ação que venha a ser proposta é conseqüência de um processo de inter-relações complexas. Este processo envolve o doente, sua rede de referência imediata e os serviços de saúde e traduz o conjunto das relações sociais vivenciadas nas distintas formas de organização da vida social, em sua dimensão global (11-13).

Nesse contexto, o objetivo do presente estudo foi caracterizar as condições existentes nos serviços de saúde públicos e filantrópicos na cidade do Recife, Brasil, para a realização do diagnóstico precoce de hanseníase, com ênfase na acessibilidade dos usuários aos serviços $(14,15)$ e em alguns indicadores indiretos da qualidade do atendimento (tempo de consulta, conhecimento clínico da hanseníase, comportamentos informados pelos médicos em relação a procedimentos para diagnóstico da hanseníase durante a consulta) (16).

\section{MATERIAIS E MÉTODOS}

O trabalho aqui descrito é um estudo de caso realizado no Recife, Brasil, de março a setembro de 1994, em uma amostra constituída por 32 serviços de saúde (tabela 1). A amostra foi selecionada a partir do grupo composto pelos 55 serviços de saúde mencionados na história pessoal de 183 pacientes de hanseníase, com idade entre 20 e 70 anos, residentes no Recife. Estes pacientes participaram de uma investigação cujo objetivo foi compreender a influência da percepção e interpretação das manifestações da doença no uso dos serviços de saúde e na adoção de outras alternativas de tratamento. Foram excluídos 23 serviços, 18 por serem privados e 5 por estarem localizados fora do Recife.

Os pacientes de hanseníase foram selecionados quando estava se consultando pela primeira vez ou completando um processo diagnóstico iniciado anteriormente, no período de novembro de 1993 a julho de 1994.

TABELA 1. Distribuição das unidades de saúde por entidade mantenedora e nível de

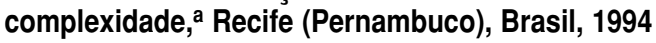

\begin{tabular}{|c|c|c|c|c|c|c|c|}
\hline \multirow{3}{*}{$\begin{array}{l}\text { Entidade } \\
\text { mantenedora }\end{array}$} & \multicolumn{6}{|c|}{ Nível de complexidade } & \multirow[b]{3}{*}{ Total } \\
\hline & \multicolumn{2}{|c|}{ Porte 1} & \multicolumn{2}{|c|}{ Porte 2} & \multicolumn{2}{|c|}{ Porte 3} & \\
\hline & No. & $\%$ & No. & $\%$ & No. & $\%$ & \\
\hline Município & 2 & 6 & 4 & 12 & - & - & 6 \\
\hline Estado & 4 & 12 & 8 & 25 & 2 & 6 & 14 \\
\hline Previdência & 1 & 3 & 3 & 9 & - & - & 4 \\
\hline Universidade & 1 & 3 & 1 & 3 & 2 & 6 & 4 \\
\hline Filantrópica & 1 & 3 & 3 & 9 & - & - & 4 \\
\hline Total & 9 & 28 & 19 & 60 & 4 & 12 & 32 \\
\hline
\end{tabular}

a Porte 1: Serviços que oferecem consultas médicas em clínicas básicas; Porte 2: serviços que oferecem consultas médicas em clínicas básicas e em alguma especialidade; Porte 3: Centros de referência para tratamento da hanseníase (serviços que oferecem consultas médicas em clínicas básicas e em alguma especialidade e contam com um serviço de dermatologia sanitária). 
Através de entrevistas semi-estruturadas, realizadas nos serviços de saúde e domicílios, explorou-se de maneira sistemática, para cada paciente, a trajetória de contatos com os serviços de saúde durante o processo diagnóstico.

Quatro instrumentos (três questionários, para gerentes, médicos e usuários, e um roteiro para observação do funcionamento) foram construídos e ajustados para coletar informações sobre as unidades de saúde utilizadas. A partir destes instrumentos, foram caracterizados: a infra-estrutura dos serviços de saúde; o modo como se desenvolvem as atividades de rotina; a facilidade de acesso à consulta; e a atitude e conhecimento dos médicos sobre hanseníase. O trabalho de campo foi executado por duas médicas, com experiência prévia em avaliação de serviços de saúde e que foram treinadas para empregar as técnicas de observação e entrevista, utilizando amostragem de etapas múltiplas.

A investigação foi realizada obedecendo três etapas, descritas a seguir: caracterização organizacional dos serviços de saúde; observação do funcionamento dos serviços de saúde; levantamento sobre a atitude e conhecimento dos médicos sobre hanseníase.

\section{Caracterização organizacional dos serviços de saúde}

Nesta etapa foram entrevistadas as pessoas responsáveis pelas atividades de gerência nas unidades de saúde, com a finalidade de: a) estabelecer o perfil das atividades oferecidas pelos serviços e o horário de atendimento; b) identificar os médicos envolvidos nas atividades de atenção ao adulto, por especialidade; c) definir o nível de complexidade de cada serviço, com base no potencial para realizar atividades relacionadas com a detecção e tratamento de casos de hanseníase; e d) caracterizar a presença de condições operacionais para realizar estas atividades.

De acordo com a capacidade potencial para executar atividades relacionadas com a detecção e tratamento da hanseníase, os serviços de saúde foram classificados como: porte 1 - serviços que oferecem consultas médicas em clínicas básicas (gineco-obstetrícia e clínica geral); porte $2-$ serviços que oferecem consultas médicas em clínicas básicas e em alguma especialidade; e porte 3 - centros de referência para tratamento da hanseníase (serviços que oferecem consultas médicas em clínicas básicas e em alguma especialidade e contam com um serviço de dermatologia sanitária). Nas unidades de porte 3 , os serviços de dermatologia sanitária responsáveis pelo diagnóstico definitivo e tratamento dos pacientes de hanseníase não foram avaliados.

\section{Funcionamento dos serviços de saúde}

Dos 32 serviços caracterizados, em 31 obteve-se autorização para investigar o funcionamento da rotina. $\mathrm{O}$ serviço onde não houve autorização foi eliminado apenas desta fase do estudo, permanecendo nas demais fases. Nesta etapa, cada serviço foi submetido a uma observação semi-estruturada em dias da semana aleatoriamente selecionados, estabelecendo uma amostragem de tempo (17) de acordo com o nível de complexidade e o número de médicos em atividade, totalizando 214 horas e 45 minutos gastos na observação dos 31 serviços.

No momento da observação, buscamos caracterizar a ocorrência de marcação prévia da consulta; o tempo de agendamento (tempo entre a marcação da consulta e sua realização); e o tempo de espera dentro da unidade (calculado como a diferença entre o momento de chegada do paciente ao serviço e o momento da entrevista). Foram entrevistados 1998 pacientes adultos que estavam esperando por uma consulta nas clínicas básicas (gineco-obstetrícia e clínica geral) e dermatológica nos serviços dos portes 1 ( $15 \%$ dos pacientes), 2 (67\%) e 3 (18\%). Procurou-se também estabelecer o tempo de duração da consulta. Entre os médicos que desenvolvem atividades em clínicas básicas e dermatologia, observou-se o tempo de duração da consulta para 123 profis- sionais $(13 \%, 73 \%$ e $14 \%$, respectivamente, dos portes 1, 2 e 3), que atenderam 1000 pacientes.

\section{Atitude e conhecimento dos médicos sobre hanseníase}

No Recife, a detecção de casos de hanseníase na rede de serviços de saúde é realizada pelos médicos. Portanto, uma caracterização exploratória da atitude e conhecimento destes profissionais em relação à doença pode fornecer pistas importantes sobre a capacidade diagnóstica dos serviços.

Para proceder a esta caracterização, o desenho do estudo estabeleceu uma amostra de 135 profissionais, calculada como um $\alpha=95 \%$, supondo uma prevalência de $10 \%$ de capacidade técnica para detectar um caso de hanseníase, e alocada em função da proporção daqueles que desenvolviam atividades em clínica geral, ginecologia e obstetrícia e dermatologia nas 32 unidades estudadas. A amostra real foi de 133 médicos.

Elaborou-se, para cada serviço, com os dados coletados na primeira etapa, uma listagem nominal por ordem alfabética dos 421 médicos envolvidos nas atividades de atenção ao adulto nas clínicas citadas. A amostra foi selecionada utilizando uma tabela de números casuais. Quando o médico selecionado estava de férias ou de licença, foi substituído através da realização de um novo sorteio. Houve apenas quatro recusas. O protocolo auto-administrado, que buscava levantar o conhecimento dos médicos sobre hanseníase, foi, em cerca de $90 \%$ dos casos, preenchido pelos médicos na presença do entrevistador.

Em função dos critérios adotados para selecionar os dias nos quais se investigou o funcionamento das unidades e para alocar os profissionais entrevistados, os médicos que foram observados em relação ao tempo de consulta não foram os mesmos que responderam o questionário. No caso dos serviços dos portes 1 e 2, entretanto, os mesmos médicos para quem se observou a duração da consulta responderam o questionário, já que estes 
portes têm menor disponibilidade de recursos humanos.

A análise foi realizada buscando: a) identificar variáveis capazes de discriminar grupos de serviços de saúde segundo o nível de complexidade e a entidade mantenedora; b) estabelecer a probabilidade de pertencer a um grupo de serviços utilizando a análise de proporções; c) explorar os intervalos de tempo (intervalo entre marcar consulta e ser atendido, tempo de espera dentro do serviço e duração da consulta) para caracterizar a mediana e os intervalos entre o primeiro e o terceiro quartis e utilizar a análise de variância de Kruskal-Wallis (18) para avaliar a existência de diferenças significativas entre os níveis de complexidade; e d) identificar a influência da especialidade e do tempo de formado na atitude e no conhecimento do médico sobre hanseníase, determinando a significância da associação através do qui-quadrado $\left(\chi^{2}\right)$ de uma amostra (18). Falamos de tendências nos resultados quando se delineiam diferenças na ausência de significância estatística.

\section{RESULTADOS}

O perfil da demanda de acordo com o acesso aos serviços de saúde - isto é, se os pacientes que procuravam o serviço de saúde eram atendidos no mesmo dia ou precisavam marcar consulta e retornar em outro dia para serem atendidos - ficou assim caracterizado: $31 \%$ dos pacientes eram atendidos no mesmo dia nos serviços do porte 1; nos serviços do porte 2, 54\% dos pacientes eram atendidos no mesmo dia; e $73 \%$ dos pacientes nos serviços de porte 3 .

Considerando o déficit no número de atendimentos em relação à demanda, de acordo com a informação administrativa, em 44, 58 e 75\%, respectivamente, das unidades dos portes 1,2 e 3, era realizada uma triagem entre os pacientes que procuravam os serviços, para estabelecer quais pacientes deveriam ter prioridade no atendimento. A observação cuidadosa da rotina destes serviços demonstrou que nos serviços do porte 1 não se rea- lizava triagem; no porte 2 , conforme nossa observação, a triagem era realizada em $12 \%$ dos serviços; e em $25 \%$ dos serviços no porte 3, apesar de todos os 31 terem apresentado demanda reprimida, ao menos em uma das amostras de tempo efetuadas.

A observação das condutas adotadas, quando da ocorrência de demanda reprimida, permitiu identificar que em apenas 9 (29\%) dos serviços estudados - 33 e $67 \%$, respectivamente, dos portes 1 e 2 , se procedeu ao agendamento dos pacientes que não conseguiram consulta no mesmo dia. Cerca de $72 \%$ dos serviços de saúde encerram o atendimento ambulatorial às 17 horas. Apenas um serviço municipal mantém-se em funcionamento até às 21.30 horas.

Excluídos os 1079 pacientes que tiveram acesso a consulta no mesmo dia, a mediana do tempo de agendamento dos demais foi maior nos serviços de saúde dos portes 1 e 3, quando comparados com os serviços do porte 2. Entretanto, análise detalhada desta série de tempo evidencia que, no porte 2, um resíduo destes pacientes demorou mais tempo entre a marcação e a consulta (figura 1). Constatou-se a existência de diferenças significativas entre os três níveis de complexidade no que diz respeito ao tempo de agendamento $(\mathrm{H}=23,727 ; P=0,000)$.

Nas unidades de saúde municipais e da previdência específica (unidades de saúde que prestam assistência médicohospitalar aos funcionários públicos estaduais e federais), com medianas do tempo de agendamento de, respectivamente, 2, 5 e 3,0 dias, uma maior proporção dos pacientes que não consultaram no mesmo dia tiveram acesso mais rápido à consulta. Nos serviços filantrópicos, a mediana do tempo de agendamento foi de 15 dias.

A figura 2 mostra que, nas unidades de saúde do porte 1 , as quais apresentaram menor facilidade de acesso no mesmo dia, observou-se a menor mediana do tempo de espera dentro do serviço. Embora os pacientes que buscaram os portes 2 e 3 tenham a mesma mediana (180 minutos), os intervalos entre o primeiro e o terceiros quartis, de 90 a 285 minutos para o porte 2 e 120 a 250 minutos para o porte 3 , mostram o comportamento distinto das duas distribuições de tempo, reforçado pela presença de um prolongamento excessivo da espera entre os

FIGURA 1. Tempo entre marcação e realização de consulta, em dias, por nível de complexidade dos serviços de saúde, Recife (Pernambuco), Brasil, 1994a



a Os serviços do porte 1 oferecem consultas médicas em clínica geral; do porte 2, consultas médicas em clínica geral e em alguma especialidade; os serviços do porte 3 são centros de referência para tratamento da hanseníase. Valores indicam tempo mínimo, mediana de tempo e tempo máximo entre marcação e realização da consulta. 
pacientes que utilizaram o porte 2 . $\mathrm{O}$ resultado da análise de variância de Kruskal-Wallis $(\mathrm{H}=36,051 ; P=0,000)$ demonstra a significância da diferença entre os portes quando se considera o tempo entre chegada ao serviço e consulta. Deve-se considerar que o tempo de espera está subestimado, na medida que foi calculado como o intervalo transcorrido desde a chegada do paciente na unidade até o momento da entrevista na sala de espera.

Embora a programação dos serviços ambulatoriais em geral estabeleça quatro consultas por hora, neste estudo a mediana do tempo de consulta foi bastante baixa, respectivamente 5,0, 6,0 e 7,0 minutos para os portes 1,2 e 3 . No entanto, uma maior proporção de pacientes que buscaram os portes 2 e 3 demoraram mais tempo no consultório médico (figura 3). Existem diferenças significativas entre os níveis de complexidade em relação ao tempo de consulta $(\mathrm{H}=10,470 ; P=0,005)$.

Quando consideradas as entidades mantenedoras, os serviços universitários, com uma mediana de tempo de consulta igual a 8,5 minutos, apresentaram o melhor desempenho. Os serviços municipais e estaduais tiveram a menor mediana de tempo de consulta (5,0 minutos), embora uma proporção discretamente maior dos pacientes atendidos nos serviços municipais tenha tido um tempo menor de permanência no consultório médico. Em todos os tipos de entidades mantenedoras e em todos os portes, foram observados pacientes que permaneceram apenas 1 minuto no consultório médico.

As deficiências na organização do sistema de pré e pós-consulta e na oferta de atividades através de outras categorias profissionais (consulta de enfermagem, serviço social, trabalho educativo), que complementariam a consulta médica, reforçam as limitações da assistência prestada.

Nas unidades do porte 1 foram encontradas as maiores deficiências em relação à disponibilidade de condições para desenvolver a detecção de casos de hanseníase, particularmente devido à ausência de recursos humanos treinados, à escassez de material especí-

FIGURA 2. Tempo de espera dos pacientes no serviço de saúde, em minutos, por nível de complexidade dos serviços, Recife (Pernambuco), Brasil, 1994a

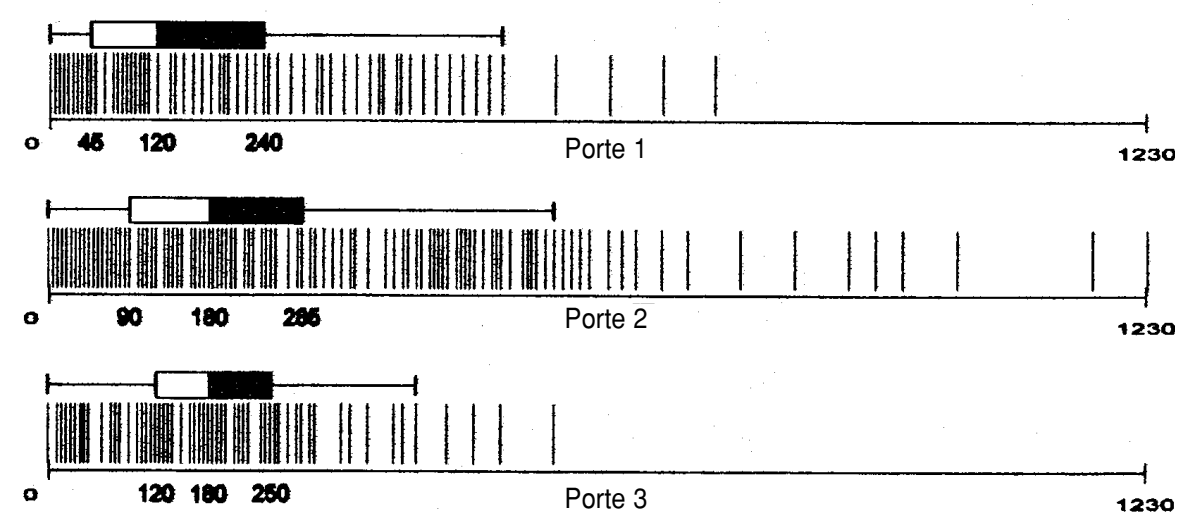

a Os serviços do porte 1 oferecem consultas médicas em clínica geral; do porte 2, consultas médicas em clínica geral e em alguma especialidade; os serviços do porte 3 são centros de referência para tratamento da hanseníase. Valores indicam tempo de espera mínimo, mediana de tempo e tempo de espera máximo.

FIGURA 3. Duração da consulta, em minutos, por nível de complexidade dos serviços de saúde, Recife (Pernambuco), Brasil, 1994a
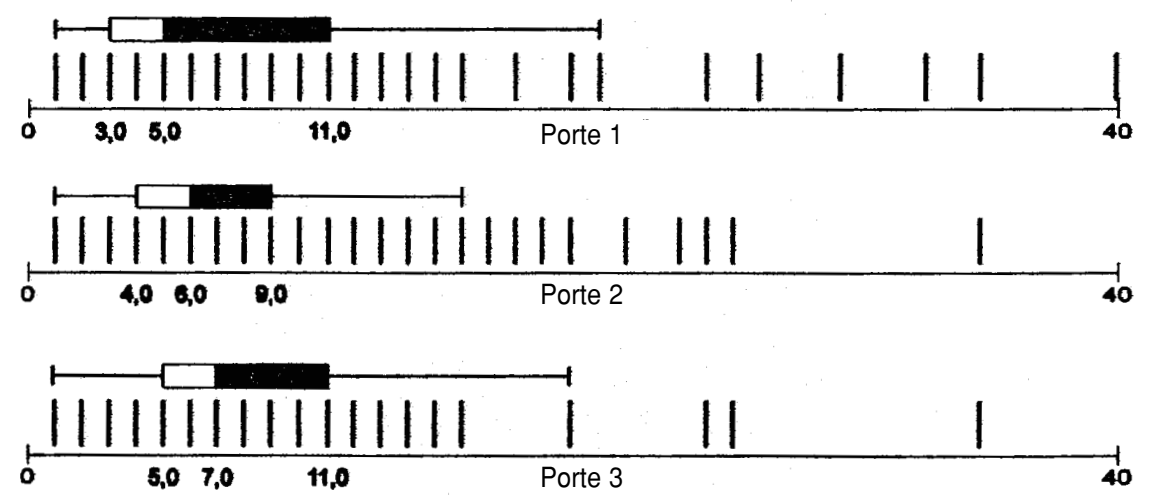

a Os serviços do porte 1 oferecem consultas médicas em clínica geral; do porte 2, consultas médicas em clínica geral e em alguma especialidade; os serviços do porte 3 são centros de referência para tratamento da hanseníase. Valores indicam tempo de espera mínimo, mediana de tempo e tempo de espera máximo.

fico para exame de sensibilidade e à subnotificação dos casos suspeitos (tabela 2). Apenas $44 \%$ de todas as unidades investigadas dispunham de algum profissional treinado para realização de diagnóstico de hanseníase.
Nos serviços filantrópicos encontraram-se os maiores obstáculos operacionais para a detecção da hanseníase.

Entre os médicos selecionados para caracterizar a atitude e os conhecimentos sobre a hanseníase, $54 \%$, $36 \%$ e $9 \%$ 
TABELA 2. Condições para o diagnóstico da hanseníase dos serviços de saúde, por nível de complexidade, ${ }^{a}$ Recife (Pernambuco), Brasil, 1994

\begin{tabular}{|c|c|c|c|c|c|}
\hline \multirow[b]{2}{*}{ Tipo de serviço } & \multicolumn{5}{|c|}{ Tipo de recurso disponível nos serviços } \\
\hline & $\begin{array}{c}\text { Manual para } \\
\text { diagnóstico clínico }\end{array}$ & $\begin{array}{l}\text { Material para exame } \\
\text { de sensibilidade }\end{array}$ & $\begin{array}{c}\text { Referência correta } \\
\text { dos casos suspeitos }\end{array}$ & Médicos treinados & $\begin{array}{l}\text { Notificação dos } \\
\text { casos suspeitos }\end{array}$ \\
\hline Porte $1(n=9)$ & $55 \%$ & $0 \%$ & $11 \%$ & $22 \%$ & $67 \%$ \\
\hline Porte $2(n=19)$ & $63 \%$ & $58 \%$ & $63 \%$ & $47 \%$ & $68 \%$ \\
\hline Porte $3(n=4)$ & $75 \%$ & $75 \%$ & $75 \%$ & $75 \%$ & - \\
\hline
\end{tabular}

a Porte 1: Serviços que oferecem consultas médicas em clínicas básicas; Porte 2: serviços que oferecem consultas médicas em clínica geral e em alguma especialidade; Porte 3: Centros de referência para tratamento da hanseníase (serviços que oferecem consultas médicas em clínicas básicas e em alguma especialidade e contam com um serviço de dermatologia sanitária).

estavam no exercício das atividades de atenção ao adulto, respectivamente, em clínica médica, ginecologia e obstetrícia e dermatologia. Do total de médicos, $15 \%$ eram do porte $1 ; 68 \%$ do porte 2 ; e $17 \%$ do porte 3 . Em relação ao tempo de formatura, 39\% dos médicos tinham se formado há 10 anos; $41 \%$, entre 11 e 20 anos; e $20 \%$ haviam se formado há mais de 20 anos. Os dermatologistas receberam treinamento específico em hanseníase.

A manifestação clínica mais citada pelos médicos para formular a hipótese diagnóstica de hanseníase foi a presença de lesão de pele com alteração da sensibilidade. Esta foi referida por $100 \%$ dos dermatologistas, independente do tempo de formatura. Cinqüenta e oito por cento dos dermatologistas citaram outros sinais no trajeto do nervo. Entre os especialistas em ginecologia e obstetrícia, $81 \%$ mencionaram a lesão de pele com alteração de sensibilidade, que entre aqueles formados há até 10 anos, representou a única manifestação mencionada. Uma menor proporção de ginecologistas (10\%) citou outros sinais no trajeto do nervo; entre os clínicos gerais, $86 \%$ citaram lesão de pele com alteração de sensibilidade, enquanto que $33 \%$ citaram outros sinais no trajeto do nervo.

Na caracterização do diagnóstico diferencial da hanseníase, a tabela 3 evidencia o melhor desempenho dos dermatologistas, apesar dos $17 \%$ que não sabiam informar como seria feito $\mathrm{O}$ diagnóstico diferencial ou que forneceram informações incorretas sobre o mesmo. Apenas $40 \%$ do total dos médicos em todas as especialidades infor- mou corretamente sobre o diagnóstico diferencial da hanseníase e, entre estes, cerca de $62 \%$ citaram apenas um, em geral, uma dermatomicose.

Quando se explora a influência do tempo de formatura na capacidade para realizar o diagnóstico diferencial, observa-se uma discreta tendência para, à medida em que aumenta o tempo de formado, diminuir a proporção de clínicos gerais com informações corretas e para aumentar a proporção daqueles com informação parcialmente correta.

Uma elevada proporção dos clínicos gerais e ginecologistas não investiga a existência de lesões de pele durante a anamnese e não examina toda a superfície corporal. Na presença de lesão suspeita de hanseníase, os clínicos gerais mostraram uma maior tendência para interrogar sobre os antecedentes da doença e pesquisar as alterações de sensibilidade. Neste contexto, os dermatologistas apresentaram um desempenho bastante diferenciado, apesar de uma proporção deles não examinar toda superfície corporal e de terem sido identificadas deficiências no enca- minhamento dos pacientes para as unidades de referência (tabela 4).

À medida em que aumenta o tempo desde a formatura, uma proporção discretamente maior dos clínicos gerais disse investigar a presença de lesões de pele durante o exame clínico de rotina, apesar da baixa freqüência de exame físico completo. Informar ao paciente a suspeita diagnóstica de hanseníase e encaminhar os casos suspeitos para as unidades de referência foi uma conduta mais freqüente entre aqueles com menor tempo de formados, embora mesmo entre estes a proporção de profissionais que encaminhava os casos suspeitos para as unidades de referência tenha sido baixa.

\section{DISCUSSÃO}

É possível que as limitações regionais do estudo tenham produzido resultados específicos a uma determinada cultura organizacional; portanto, é preciso ter cautela no que diz respeito à generalização dos dados. Mesmo

TABELA 3. Conhecimento dos médicos para realizar diagnósticos diferenciais da hanseníase, segundo especialidade, Recife (Pernambuco), Brasil, 1994

\begin{tabular}{|c|c|c|c|c|c|c|c|c|}
\hline \multirow[b]{3}{*}{ Especialidade } & \multicolumn{8}{|c|}{ Informações para realizar diagnóstico diferencial } \\
\hline & \multicolumn{2}{|c|}{ Corretas } & \multicolumn{2}{|c|}{$\begin{array}{l}\text { Parcialmente } \\
\text { corretas }\end{array}$} & \multicolumn{2}{|c|}{ Incorretas } & \multicolumn{2}{|c|}{ Não sabe } \\
\hline & No. & $\%$ & No. & $\%$ & No. & $\%$ & No. & $\%$ \\
\hline Clínico geral $(n=66)$ & 29 & 44 & 15 & 23 & 14 & 21 & 8 & 12 \\
\hline Ginecologista-obstetra $(n=43)$ & 14 & 32 & 2 & 5 & 19 & 44 & 8 & 19 \\
\hline Dermatologista $(n=12)$ & 5 & 42 & 5 & 42 & 1 & 8 & 1 & 8 \\
\hline
\end{tabular}


TABELA 4. Comportamento informado pelos médicos em relação a procedimentos para diagnóstico de hanseníase durante a consulta, segundo especialidade, Recife (Pernambuco), Brasil, 1994



assim, acreditamos que as recomendações para orientar o processo de diagnóstico precoce de hanseníase transcendam o âmbito local.

Foram muitas as dificuldades apresentadas pelos serviços para efetivar o atendimento. Entre elas, presença sistemática de demanda reprimida, independente da utilização (ou não) de critérios de risco para selecionar os indivíduos mais necessitados de consulta nos pontos de acesso ao sistema de saúde (apesar das limitações do enfoque, a triagem continua sendo uma atividade importante para a prática clínica); processo deficiente de organização da demanda; grande tempo de agendamento; e longas esperas dentro das unidades de saúde.

Os serviços do porte 1, porta de entrada do sistema de saúde, foram os que apresentaram menor proporção de pacientes com consulta no mesmo dia. A inconsistência encontrada entre as duas fontes de informação utilizadas neste estudo (informação administrativa e observação), em relação à realização de triagem, sugere que alguns serviços consideraram como sistemática uma triagem que talvez seja executada de forma esporádica e ocasional. Em relação ao grande número de pacientes que não conseguiam ser atendidos no mesmo dia em que procuravam os serviços de saúde, o que se evidencia aqui é a profunda defasagem entre a demanda de grupos específicos da população e a organização institucional. Em termos de horário de atendimento, a baixíssima oferta de atividades no período noturno pode se constituir em um importante obstáculo para o acesso da população trabalhadora.

Por outro lado, a curta duração das consultas, as características do exame clínico de rotina, o generalizado déficit de condições operacionais para detectar casos de hanseníase, as deficiências observadas no reconhecimento clínico da doença e a baixa proporção de médicos das clínicas básicas que receberam treinamento específico, evidenciam as sérias limitações do sistema de saúde no manejo do diagnóstico precoce da hanseníase. Cabe ressaltar que os problemas técnicos e operacionais observados nos serviços de porte 3, não dizem respeito ao setor de dermatologia sanitária, mas ao processo de atendimento desenvolvido pelos clínicos gerais, ginecologistas e dermatologistas que consultam a demanda geral de adultos. No entanto, os resultados parecem surpreendentes, uma vez que se poderia esperar um melhor desempenho desses serviços.

Nosso estudo identificou a persistência de fortes obstáculos para a detecção precoce da hanseníase. A realidade dos serviços é preocupante, as sérias deficiências reveladas têm raízes nos problemas organizacionais e administrativos que afetam a prestação adequada da atenção em geral $(8,14,15)$ e na posição periférica da hanseníase em uma situação histórica concreta (10).

Nossos resultados apontam para a necessidade de interpretar de forma mais cautelosa o comportamento das estimativas, estabelecidas utilizando dados de registro, sob o risco de, ao menos no caso específico do Recife, subdimensionar a cadeia de transmissão da doença. Segundo Lechat et al. (19), não se deve atribuir a estas estimativas um valor excessivo; elas devem ser questionadas e revisadas à medida em que se adquire experiência e informação.

Considerando que a doença traduz as inter-relações entre os processos corporais e as categorias sócioculturais, entre experiência e significado, o manejo de uma doença como a hanseníase compete com outros riscos, necessidades e racionalidades, que podem resultar mais urgentes para os pacientes. Portanto, os obstáculos encontrados podem estimular o adiamento do retorno aos serviços de saúde, facilitando o desenvolvimento ou agravamento de incapacidades provocadas pela hanseníase e a persistência da transmissibilidade.

Desde que se pretenda viabilizar o propósito da Organização Mundial da Saúde (OMS) (20) de eliminar a hanseníase como um problema de saúde pública, torna-se uma tarefa urgente a capacitação de recursos humanos, com simultânea reestruturação da dinâmica funcional dos serviços de saúde, para detectar, encaminhar e tratar estes pacientes, executando as ações básicas de vigilância epidemiológica pertinentes ao processo. Ademais, necessita-se conhecer as interpretações e alternati- 
vas que resultam da realidade objetiva do cotidiano nos grupos a quem a ação de controle se dirige $(12,21)$. Para Kadt e Tasca (22), as intervenções que visam a aperfeiçoar a operação e fornecimento de serviços de saúde podem até nes RAA. A expansão da hanseníase no nordeste brasileiro. Rev Saude Publ 1989;23: 101-116.

2. Pernambuco, Secretaria de Saúde de Pernambuco. Relatório anual das ações de controle da hanseníase em 1992. Recife: Secretaria de Saúde de Pernambuco; 1992. (Documento mimeografado).

3. World Health Organization. Expert Committee on Leprosy: sixth report. Geneva: WHO; 1988. (Technical Report Series No 768).

4. World Health Organization. A guide to leprosy control. 2nd ed. Geneva: WHO; 1988.

5. Yekutiel P. Lecciones que se derivan de las grandes campañas de erradicación. Foro Mundial Salud 1981;2:141-170.

6. Lechat MF. Control programs in leprosy. Em: Hastings RC, Convit J. Leprosy. New York: Churchill Livingstone; 1985:167-192.

7. Brasil, Ministério da Saúde. Plano de eliminação da hanseníase no Brasil para o período de 1995-2000. Brasília: Fundação Nacional de Saúde/CENEPI/Coordenação Nacional de Dermatologia Sanitária; 1995.

8. Carvalho MR. Modelos assistenciais de unidades básicas e o conceito de integralidade. Em: Bodstein R. Serviços locais de saúde: cons- ajudar a reduzir iniqüidades, desde que envolvam critérios rigorosos para definir as prioridades, pois serão mais importantes quanto mais forem dirigidas para os grupos que vivem em condições mais desfavoráveis.

\section{REFERÊNCIAS}

trução de atores e políticas. Rio de Janeiro: Relume-Dumará; 1993:117-130.

9. Sevilla E. Mutilados del oprobio. Bogotá: Tercer Mundo Editores/Colcultura; 1995.

10. Lessa ZL. Doença e estigma. Cad Hist Saude (Rio de Janeiro) 1992;2:17-21.

11. Infante-Castañeda C. Bases para el estudio de la interacción familia-redes sociales: uso de servicios de salud. Salud Publica Mex 1988;30: 175-196.

12. Minayo MCS. Abordagem antropológica para avaliação de políticas sociais. Rev Saude Publ 1991;25:233-238.

13. Feliciano KVO, Kovacs MH. Opiniões sobre a doença entre membros da rede social de pacientes de hanseníase no Recife. Rev Panam Salud Publica 1997;1:112-118.

14. Vellozo VRO, Souza RG. Acesso e hierarquização: um caminho (re)construído. Em: Bodstein R. Serviços locais de saúde: construção de atores e políticas. Rio de Janeiro: RelumeDumará, 1993:97-116.

15. Cohn A, Nunes E, Jacobi PR, Karsch US. O acesso em discussão: o viés da racionalidade e o viés da carência. Em: A saúde como direito e como serviço. São Paulo: Cortez Editores; 1991: 67-94.

16. Azevedo AC. Indicadores de qualidade $\mathrm{e}$ produtividade em serviços de saúde. Rev Indi-
Agradecimentos. Agradecemos à Dra. Dionila Ferreira de Andrade e à Dra. Ceres Gonçalves Machado pela realização do trabalho de campo. cadores Qualidade Produtividade IPEA 1994;2: 49-55.

17. Triviños ANS. Introdução à pesquisa em ciências sociais: a pesquisa qualitativa em educação. São Paulo: Editora Atlas; 1992.

18. Siegel S. Estadística no paramétrica aplicada a las ciencias de la conducta. México, DF: Editora Trillas, 1979.

19. Lechat MF, Mission CB, Sansarricq H, Declercq E, Vanderveken M. Utilización de los datos. Em: Sistema de registro y notificación de casos de lepra. Genebra: WHO; 1987:65-87

20. World Health Organization. La lepra tiene los días contados. Foro Mundial Salud 1991;12:30.

21. Slovic P. Perception of risk. Science 1987;236: 280-285.

22. Kadt E, Tasca R. Enfrentando a iniqüidade: estratégias de intervenção que enfocam chances de saúde. Em: Promovendo a eqüidade de um novo enfoque com base no setor da saúde. São Paulo-Salvador: Hucitec/Cooperação Italiana em Saúde; 1993:31-43.

Manuscrito recebido em 21 de fevereiro de 1992. Aceito em versão revisada em 18 de fevereiro de 1998.

ABSTRACT This paper presents the results of a descriptive study carried out in the city of Recife, state of Pernambuco, Brazil, between March and September 1994. The study aimed at health services available for performing early diagnosis of Hansen's disease with emphasis on accessibility and quality of the services provided. The sample consisted of 32 health clinics visited for diagnostic purposes by 183 patients with Hansen's disease. Information on organizational infrastructures was collected by means of interviews with health clinic managers. Information regarding routine procedures in the 32 clinics was collected by observation, with special attention given to archival and inspection activities. A total of 1998 patients were interviewed to determine accessibility of services. Time spent in consultation with the physician was determined for 1000 patients who were seen by 123 physicians at the clinics during the interviews. To explore physicians' attitude and knowledge regarding Hansen's disease, 133 were randomly selected from a list of names.

The following factors were identified as hindering early diagnosis of Hansen's disease: the large number of people seeking service who could not be seen by a physician on the same day; the long time elapsed between appointment scheduling and the actual visit (for those not seen on the same day); the long wait for the consultation; the brevity of the consultation; the low availability of trained personnel; the low proportion of physicians who examined all body surfaces; difficulties in the clinical recognition of the disease; and physicians not prepared to make a differential diagnosis. These obstacles can precipitate the physical deterioration of Hansen's disease patients and stimulate the persistence of transmissibility; therefore, they need to be overcome if Hansen's disease is to be eliminated. 